\title{
Vorausberechnungen der schweizerischen Wohnbevölkerung
}

G. Schumacher

\section{Einleitung}

Verschiedene Anregungen leisten heute immer wieder dazu Vorschub, sich mit Vorausberechnungen von Bevölkerungszahlen auseinanderzusetzen. Die wichtigsten von ihnen liegen in geographischen, planerischen und volkswirtschaftlichen Tätigkeitsbereichen.

Vor allem stellen sich diese heiklen Fragen stets in der Orts-, Regional- und Landesplanung, da für die Entwicklungs- und Ausbaupläne eine wenigstens annähernd begründbare Basis vorliegen sollte. Die Frage also, die sich immer wieder stellt, lautet etwa wie folgt: Mit wieviel Einwohnern haben wir zu diesem oder jenem Zeitpunkt zu rechnen? In der Regel ist es leichter für ein größeres Gebiet Prognosen zu erarbeiten als für ein kleineres. Daher soll sowohl die gesamte Fläche wie auch die Wohnbevölkerung der Schweiz in diesem Rahmen relevant sein. Von der gesamten Wohnbevölkerung lassen sich dann die Beziehungen zu den Kantonen, Regionen und eventuell sogar bis zu den Gemeinden verfolgen.

Diese Zusammenstellung verfolgt zwei Ziele. Einmal soll sie eine Übersicht und eine Auswahl von Vorausberechnungen, die die Schweiz betreffen, sein, um einerseits etwa gleichlautende und anderseits auch divergierende Resultate aufzuzeigen. Dann soll sie auch für jene, die sich mit Vorausberechnungen kleinerer Größen von Wohnbevölkerungszahlen innerhalb der Schweiz befassen, gewisse Bezugsgrößen anbieten.

Die geradlinige Fortschreibung nach den Angaben des Statistischen Büros des Kantons Bern für die Bevölkerungszunahme der Schweiz

In den Mitteilungen (Nr. 45) des Statistischen Büros des Kantons Bern finden sich verschiedene generelle Hinweise bezüglich einer Bevölkerungsvermehrung und auf die Zahlen einer geradlinigen Fortschreibung bis zum Jahre 1990 der Wohnbevölkerung der Schweiz. Es handelt sich hier nicht direkt um eine eigentliche Prognose, sondern vielmehr um eine einfach durchgeführte geradlinige Fortschreibung. Auf deren Grundlage ergeben sich folgende Zahlen:
Jahr Wohnbevölkerung der Schweiz

1960

5429000

1970

6100000

1980

6800000

1990

7300000

Eidgenössisches Statistisches Amt (EStA):

Entwicklung der Wohnbevölkerung der Schweiz 1964-1980

(Biometrische Methode, Dr. E. Hülsen)

Das Eidgenössische Statistische Amt hat nach den Weisungen der OCDE eine Bevölkerungsvorausberechnung nach der biometrischen Methode (Stichtag 1. Januar 1964) bis zum Jahre 1980 durchgeführt. Die wichtigsten Ergebnisse dieser Berechnung sind folgende:

Geschätzte Wohnbevölkerung der Schweiz 19641980. Schweizer und Niedergelassene sowie kontrollpflichtige Ausländer (ohne Wanderung)

Ausgangsbevölkerung 1.1.1964: 5717800

Jahr Wohnbevölkerung der Schweiz

$\begin{array}{ll}1970 & 6000700 \\ 1975 & 6219500 \\ 1980 & 6398500\end{array}$

Bevölkerungsentwicklung der Schweiz (Prof. Dr. W. Bickel)

Die Extrapolation der Trendwerte nach den Ausgangszahlen der Volkszählungen 1850, 1880, 1900 ergeben für das Jahr 2000:

$\begin{array}{cccc}\text { Jahr } & \begin{array}{c}\text { VZ 1850 } \\ \text { Ausgangszahl }\end{array} & \begin{array}{c}\text { VZ 1880 } \\ \text { Ausgangszahl }\end{array} & \begin{array}{c}\text { VZ } 1900 \\ \text { Ausgangszahl }\end{array} \\ 1850 & 2393000 & - & - \\ 1880 & 2832000 & 2832000 & - \\ 1900 & 3315000 & 3315000 & 3315000 \\ 1960 & 5429000 & 5429000 & 5429000 \\ 1970 & 5522000 & 5609000 & 5503000 \\ 1980 & 5936000 & 6054000 & 5971000 \\ 1990 & 6382000 & 6534000 & 6421000 \\ 2000 & 6861000 & 7052000 & 6905000\end{array}$


W. Bickel rechnet nicht mit einem gleich starken Anwachsen der Bevölkerung für die kommende Periode, da vor allem der Bevölkerungszuwachs im Jahrzehnt 1950-1960 auf einen zu großen Wanderungsgewinn zurückzuführen ist. Würde man in Zukunft mit einer gleichstarken Zunahme rechnen, so müßte die Schweiz im Jahre 2000 etwa 8285000 Einwohner haben, wovon 1773000 oder 21,4\% Ausländer wären.

\section{Rechnungsgrundlagen in der $A H V$ (Dr. K. Fricker)}

Obwohl die für die Berechnungen angenommenen Wohnbevölkerungszahlen der AHV (Alters- und Hinterbliebenenversicherung) eigentlich nicht Anspruch darauf erheben, eine Vorausberechnung zu sein, so können sie doch einen Hinweis auf Möglichkeiten der künftigen Bevölkerungsentwicklung bieten. Für die AHV bilden die errechneten Bevölkerungszahlen Modellzahlen, mit denen die Ausgaben der Versicherung gemessen bzw. budgetiert werden können.

Zunächst hatte das Bundesamt für Sozialversicherung zwei Modelle, eine statische und eine dynamische Variante, berücksichtigt. Aus der Kombination dieser beiden Modelle, welche offensichtlich Extremwerte lieferten, entstand das Modell einer semidynamischen Variante, deren Ergebnisse wie folgt lauten:
Entwicklung der schweizerischen Wohnbevölkerung (AHV)

Jahr Wohnbevölkerung Prozentuale Zuwachsrate

$\begin{array}{lll}1960 & 5429061 & 2,23 \\ 1961 & 5560000 & 2,41 \\ 1965 & 5970783 & 1,89 \\ 1970 & 6395342 & 1,12 \\ 1975 & 6712021 & 0,97 \\ 1985 & 7138535 & 0,51 \\ 2000 & 7645575 & 0,48\end{array}$

Vorausberechnungen der Wohnbevölkerung im Rahmen der Prognosenkommission der Schweizerischen Statistischen Ämter

Dr. E. Hülsen (EStA) und Dr. K. Fricker (AHV) haben neben den vorgenannten Berechnungen der Wohnbevölkerung im Rahmen der eidgenössischen Prognosenkommission weitereVorausberechnungen unternommen. Dabei wurden die durchschnittlichen Vermehrungsraten der Zeitperioden 1900-1960, $1930-1960$ und $1950-1960$ berücksichtigt. Beim Vergleich der errechneten Werte für das Jahr 2000 mit dem Ergebnis des semidynamischen Modells tritt deutlich hervor, daß die Variante der Periode 1930 - 1960 und das semidynamische Modell in ihrem Ergebnis in bezug auf das Jahr 2000 sich ziemlich nahekommen. Jedoch sind die Wachstumsvorgänge zwischen 1960 und 1990 ziemlich verschieden. Da das semidynamische Modell der Bevölkerungszahl von 1965 ungefähr entspricht, dürfte auch diesem und seinen bezüglichen Wachstumsraten eine größere Wahrscheinlichkeit zukommen.

Die drei Varianten aufgrund der Wachstumsraten der Perioden 1900-1960, 1930 - 1960 und 19501960 sind folgende:

\begin{tabular}{|c|c|c|c|c|}
\hline Jahr & $\begin{array}{l}\text { Wohnbevölkerung } \\
\text { nach VZ }\end{array}$ & $\begin{array}{l}\text { 1. Variante } \\
\text { Basis } 1900-1960\end{array}$ & $\begin{array}{l}\text { 2. Variante } \\
\text { Basis } 1930-1960\end{array}$ & $\begin{array}{l}\text { 3. Variante } \\
\text { Basis } 1950-1960\end{array}$ \\
\hline 1900 & 3315443 & 3315000 & - & - \\
\hline 1910 & 3753293 & 3572000 & - & - \\
\hline 1920 & 3880320 & 3849000 & - & - \\
\hline 1930 & 4066400 & 4147000 & 4066000 & - \\
\hline 1941 & 4265703 & 4468000 & 4469000 & - \\
\hline 1950 & 4714992 & 4815000 & 5828000 & 4715000 \\
\hline 1960 & 5429061 & 5187000 & 5261000 & 5429000 \\
\hline 1970 & . & 5590000 & 5733000 & 6251000 \\
\hline 1980 & . & 6023000 & 6247000 & 7197000 \\
\hline 1990 & . & 6490000 & 6808000 & 8287000 \\
\hline 2000 & . & 6993000 & 7418000 & 9542000 \\
\hline
\end{tabular}


Millionen Wohnbevölkerung

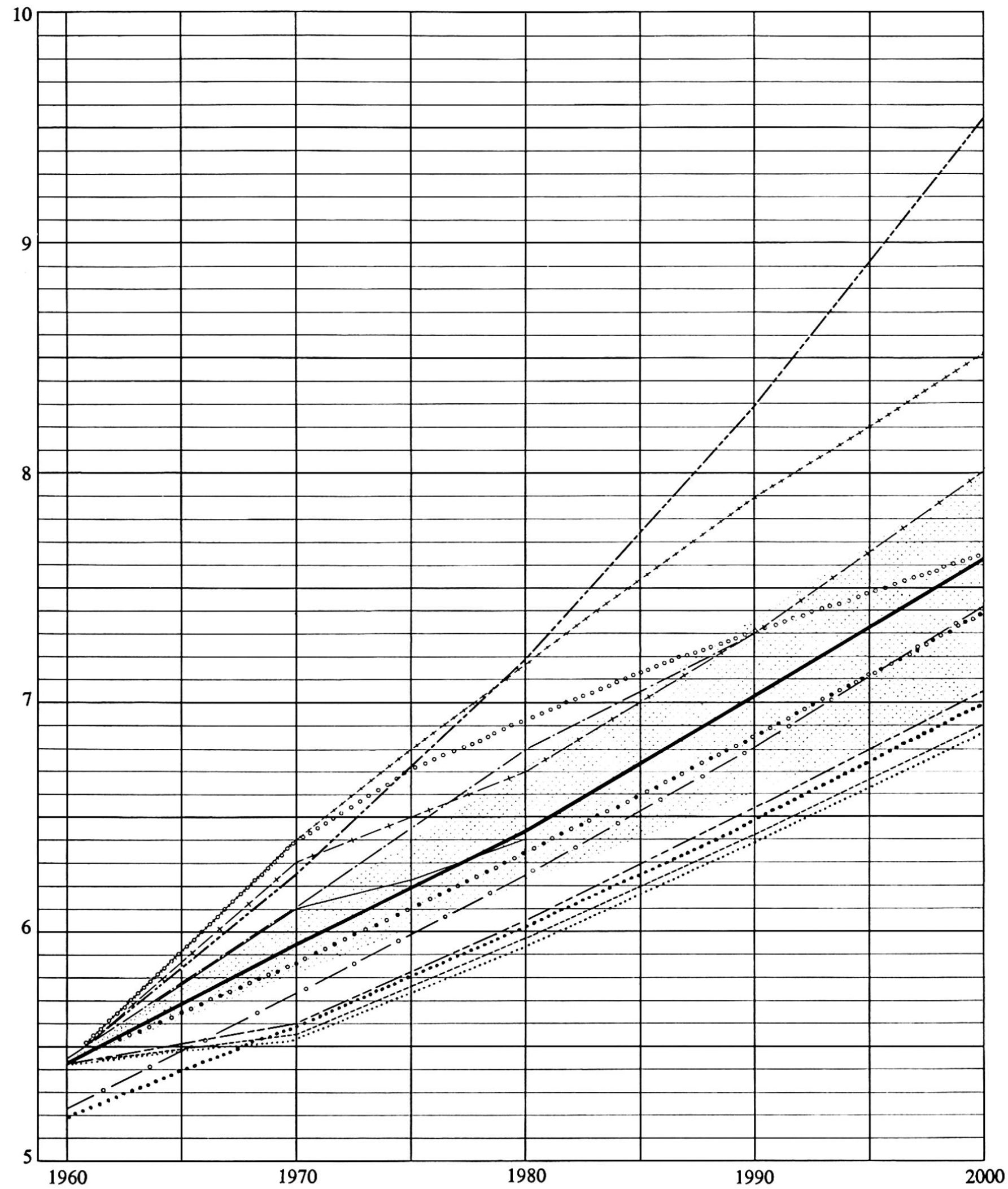

Stat. Büro Kt. Bern

ESTA (Biometrische Methode)

Bickel: Ausgang Volkszählung 1850

Bickel: Ausgang Volkszählung 1880

Bickel: Ausgang Volkszählung 1900

Fricker Semi-dyn. Modell

Fricker-Hülsen Wachstumsrate

1900 I. Variante
_•_ Fricker-Hülsen Wachstumsrate 1930 II. Variante

---_ Fricker-Hülsen Wachstumsrate 1950 III. Variante Kommission Schweiz. Stat. Ämter

.... Studienstadt im Furttal

+++ PTT

-+- ORL

— Gemittelte Werte 
Veröffentlichung der Kommission des Verbandes der Schweizerischen Statistischen Ämter zur Beurteilung der Bevölkerungsentwicklung:

Bickel, Fricker, Hülsen, Messmer

Die Kommission veröffentlichte die Aufsätze zur Vorausberechnung von Prof. Dr. W. Bickel, Dr. E. Hülsen und Dr. K. Fricker unter dem Titel «Zur mutmaßlichen Bevölkerungsentwicklung in der Schweiz. Im Vorwort dieser Veröffentlichung finden sich folgende koordinative Bemerkungen: «Die Kommission hatte sich zuerst über die Methode ihrer Arbeit schlüssig zu werden. Es zeigte sich, daß der Anteil der Ausländer an der Bevölkerung der Schweiz ein Beurteilungskriterium darstellt, das anderen Bedingungen unterliegt als die einheimische Bevölkerung. So äußern sich die Altersstruktur der Fremdarbeiter und die damit zusammenhängenden bevölkerungsmäßigen Auswirkungen zum Beispiel darin, daß sich hier eine Komponente findet, die dem Ưberalterungstrend der Schweizer entgegenwirkt. Es ist wesentlich für die Beurteilung der Bevölkerungsentwicklung, abzuschätzen, wie lange und wie stark diese Wirkung anhalten wird. Daneben haben Assimilierungsfaktoren, wie die Möglichkeit der Niederlassung und Einbürgerung, zusammen mit der spezifischen Fruchtbarkeit der Ausländer Einfluß auf die Bevölkerungszahl. Neben den natürlichen Ursachen der Bevölkerungsentwicklung spielen die wirtschaftlichen, sozialen, politischen, psychologischen und weltanschaulichen Gegebenheiten für die Zahl der Bevölkerung eine wesentliche Rolle.

Es ist nicht möglich, die Veränderung der heutigen Verhältnisse auf allen diesen Gebieten in der $\mathrm{Zu}$ kunft vorauszusehen, weshalb der Beurteilung nur gewisse richtungsweisende Annahmen zugrunde gelegt werden können.

Die Kommission kam zum Schluß, daß für das Jahr 2000, je nach Entwicklung des Ausländerbestandes, eine Einwohnerzahl von 7 bis 8 Millionen zu erwarten ist. Eine Einwohnerzahl von 10 Millionen in einem halben Jahrhundert (1960-2010) käme nur dann in Frage, wenn die Zahl der Fremdarbeiter bis dahin auf 2 bis 2,5 Millionen ansteigen würde. Jedenfalls lassen Fruchtbarkeit und Sterblichkeit der einheimischen Bevölkerung - Schweizer und niedergelassene Ausländer — nicht auf ein entsprechendes Wachstum schließen.»

Bevölkerungsprognose in: "Die Studienstadt im Furttal»

Die Autoren dieser Publikation rechnen mit dem Abklingen des starken Bevölkerungszuwachses (u.a. Einwanderungsstop). Der durchschnittliche Bevöl- kerungszuwachs wird mit $0,8 \%$ im Jahr veranschlagt. Danach ergibt sich folgende Prognose für die Einwohnerzahl der Schweiz:

$\begin{array}{ll}\text { Jahr } & \text { Einwohner } \\ 1960 & 5429000 \\ 1970 & 5863000 \\ 1980 & 6332000 \\ 1990 & 6839000 \\ 2000 & 7386000\end{array}$

\section{Die Vorausberechnung der PTT}

Die PTT rechnen bis zum Jahre 2000 mit einer durchschnittlichen jährlichen Zunahme der Bevölkerung von 1,17\%. Entsprechend dieser Annahme hätte die Schweiz im Jahre 2000 etwa 8,52 Mio Einwohner. Die einzelnen Bevölkerungszahlen sind demnach:

$\begin{array}{ll}\text { Jahr } & \text { Einwohner } \\ 1970 & 6400000 \\ 1975 & 6800000 \\ 1980 & 7170000 \\ 1985 & 7530000 \\ 1990 & 7890000 \\ 1995 & 8200000 \\ 2000 & 8520000\end{array}$

Jedoch muß in diesem Zusammenhang beigefügt werden, daß die PTT die Wohnbevölkerungszahl bewußt an der oberen Grenze angesetzt haben, da sie mit einer gewissen größeren Marge rechnen müssen, die vor allem auf Ausbaugrade ihrer Bauten und Dienste Rücksicht und Voraussicht zu nehmen hat.

Untersuchung über das Wachstum der schweizerischen Wohnbevölkerung bis zum Jahre 2000

(Zwischenbericht zur Industriestandortstudie des Institues für Orts-, Regional- und Landesplanung der ETH; Verfasser R. Schraner)

Diese Untersuchung hat von vorneherein auf mathematische Berechnungen verzichtet. Angewendet wurden verschiedene Trendextrapolationen. Die Vorausberechnungen wurden für Schweizer und Ausländer getrennt durchgeführt. Für die Berechnung der Schweizer diente die graphische Extrapolation, während für die Berechnung der Ausländer wirtschaftliche, soziale und politische Entwicklungsfaktoren berücksichtigt wurden.

Es wurde festgehalten, daß die Vermehrung der in der Schweiz heimatberechtigten Bevölkerung (Schweizer) ziemlich regelmäßig erfolgt und keinen 
großen Schwankungen unterliegt: «Untersuchungen haben gezeigt, daß die Mentalität der Bevölkerung in bezug auf Fortpflanzung und Familienbildung keinen wesentlichen Änderungen zustrebt. Sowohl effektive durchschnittliche Familiengröße wie gewünschte Kinderzahl innerhalb einer Familie liegen sehr nahe beieinander und ergeben für die Schweiz knapp drei Kinder ... Daneben steht fest, daß eine Kinderzahl von 2,5 im Durchschnitt notwendig ist, um eine bestimmte Bevölkerung auf ihrem zahlenmäßigen Fortbestand zu erhalten. Was darüber hinaus geht, dient der Vermehrung. Man rechnet bei einer durchschnittlichen Kinderzahl von 3 je Familie mit rund 80 bis 100 Jahren für eine Verdoppelung der Bevölkerung. Die Zahl der Schweizer wird daher in den nächsten Jahrzehnten konstant in relativ mäßigem Tempo wachsen.»

Der Anteil der Ausländer wird wie folgt eingeschätzt: rund $15 \%$; also Schweizer und etwa $15 \%$ Ausländer $=$ Wohnbevölkerung der Schweiz. Das Ergebnis der normalen Extrapolation für die Schweiz plus die Einschätzung der Ausländer ist folgendes:

$\begin{array}{lllll}\text { Jahr } & \text { Schweizer } & \text { Wohnbevölkerung } \\ 1960 & & & 5,43 \text { Mio } & \\ 1970 & 5,3 \text { Mio } & 1 \text { Mio } & 6,3 \text { Mio } & 870000 \\ 1980 & 5,7 \text { Mio } & 1 \text { Mio } & 6,7 \text { Mio } & 400000 \\ 1990 & 6,2 \text { Mio } & 1,1 \text { Mio } & 7,3 \text { Mio } & 600000 \\ 2000 & 6,8 \text { Mio } & 1,2 \text { Mio } & 8,0 \text { Mio } & 700000\end{array}$

Zusammenfassende Übersicht der angeführten Vorausberechnungen für die Wohnbevölkerung der Schweiz

Alle Bevölkerungszahlen in Millionen

$\begin{array}{rlllllllll} & 1960 & 1964 & 1970 & 1975 & 1980 & 1985 & 1990 & 1995 & 2000 \\ 1 & 5,429 & - & 6,100 & - & 6,800 & - & 7,300 & - & - \\ 2 & - & 5,718 & 6,100 & 6,220 & 6,400 & - & - & - & - \\ 3 & 5,429 & - & 5,522 & - & 5,936 & - & 6,382 & - & 6,861 \\ 4 & 5,429 & - & 5,609 & - & 6,054 & - & 6,534 & - & 7,052 \\ 5 & 5,429 & - & 5,553 & - & 5,971 & - & 6,421 & - & 6,905 \\ 6 & 5,429 & - & 6,395 & 6,712 & - & 7,139 & - & - & 7,646 \\ 7 & 5,187 & - & 5,590 & - & 6,023 & - & 6,490 & - & 6,993 \\ 8 & 5,261 & - & 5,733 & - & 6,247 & - & 6,808 & - & 7,418 \\ 9 & 5,429 & - & 6,251 & - & 7,197 & - & 8,287 & - & 9,542 \\ 10 & 5,429 & - & - & - & - & - & - & - & 7,0-8,0 \\ 11 & 5,429 & - & 5,863 & - & 6,332 & - & 6,839 & - & 7,386 \\ 12 & 5,429 & - & 6,400 & 6,800 & 7,170 & 7,350 & 7,890 & 8,200 & 8,520 \\ 13 & 5,430 & - & 6,300 & - & 6,700 & - & 7,300 & - & 8,000 \\ 14 & 5,429 & - & 5,950 & - & 6,440 & - & 7,025 & - & 7,620\end{array}$

$1=$ Stat. Büro des Kantons Bern

$2=$ EStA (Biometrische Methode)

3 = Bickel, Ausgang von Volkszählung 1850

$4=$ Bickel, Ausgang von Volkszählung 1880

$5=$ Bickel, Ausgang von Volkszählung 1900

$6=$ Fricker (AHV), semidynamisches Modell

$7=$ Fricker-Hülsen Wachstumsraten 1900: 1. Variante 5,187

$8=$ Fricker-Hülsen Wachstumsraten 1930: 2. Variante 5,261

$9=$ Fricker-Hülsen Wachstumsraten 1950: 3. Variante 5,429

$10=$ Kommission: Schweizerische Statistische Ämter

$11=$ Studienstadt im Furttal

$12=$ PTT

13 = Institut für Orts-, Regional- und Landesplanung (ORL), ETH

$14=$ Gemittelte Werte (unter Ausschluß der Extremwerte) 
Nach dieser Ưbersichtstabelle können die verschiedenen Ergebnisse der hier berücksichtigten Vorausberechnungsarten für die Schweiz im Jahre 2000 in vier Gruppen aufgeteilt werden. Die erste Gruppe der Ergebnisse (4 Berechnungsarten) liegt bei 7 Mio Einwohnern (Bickel: Ausgangszahlen VZ 1850, VZ 1880, VZ 1900 und Fricker-Hülsen Wachstumsrate 1900,1 . Variante), die zweite liegt bei 7,5 Mio Einwohnern mit vier Berechnungsarten (Fricker semidynamisches Modell; Fricker-Hülsen Wachstumsrate 1930, 2. Variante; "Studienstadt im Furttal» und das gewogene Mittel der Kommission der Schweizerischen Statistischen Ämter), die dritte Gruppe liegt bei 8,0-8,5 Mio Einwohner mit zwei Berechnungsarten (PTT und ORL) und eine vierte Gruppe mit 9,5 Mio Einwohnern mit nur einer Berechnungsart (Fricker-Hülsen Wachstumsrate 1950, 3. Variante).

Wenn nun bei kantonalen, regionalen oder eventuell sogar kommunalen Vorausberechnungen nicht auf nur eine der Berechnungsarten der schweizerischen Wohnbevölkerung für das Jahr 2000 Bezug genommen werden möchte, so können die gemittelten Werte der verschiedenen Prognosen sicherlich gewisse Anhaltspunkte anbieten.

\section{Literatur}

1. Die Studienstadt im Furttal. 2. Bericht. Wirtschaftliche, rechtliche und organisatorische Fragen. FGS. Zürich 1963.

2. EStA: Entwicklung der Wohnbevölkerung der Schweiz 1964-1980. In Heft 3 «Die Volkswirtschaft», 1965.

3. Kommission zur Beurteilung der Bevölkerungsprognosen: Demographische Rechnungsgrundlagen in der AHV. (Entwurf) 25. 5. 1964.

4. Mitteilungen des Statistischen Büros des Kantons Bern, Nr. 45: Familienstatistik und Bevölkerungsbewegung. Genealogische Stichproben in fünf Bürgerschaften. Bern 1962.

5. Schraner, R.: Untersuchung über das Wachstum der schweizerischen Wohnbevölkerung bis zum Jahr 2000. Zwischenbericht Nr. 3 der Industriestandortstudie des ORL-Institutes der ETH. Zürich 1964.

6. Schweizerische Zeitschrift für Volkswirtschaft und Statistik: Zur mutmaßlichen Bevölkerungsentwicklung in der Schweiz (Sonderdruck).

7. Statistisches Bundesamt Wiesbaden. Bevölkerung und Kultur; Sonderbeiträge: Vorausschätzung der Bevölkerung für die Jahre 1964-2000. Dezember 1963, Wiesbaden. 